\title{
Formation of physical-gel redox electrolytes through self-assembly of discotic liquid crystals: applications in dye sensitized solar cells
}

\author{
Ammar A. Khan ${ }^{a^{*}}$, Muhammad A. Kamarudina, Malik M. Qasimª Timothy D. \\ Wilkinson $^{a}$ \\ ${ }^{a}$ Centre of Molecular Materials for Photonics and Electronics, Department of Engineering, Electrical Engineering \\ Division, University of Cambridge, 9 J.J. Thomson Avenue, Cambridge, CB3 0FA, UK Email: aak46@cam.ac.uk
}

\begin{abstract}
The self-assembly of small molecules into ordered structures is of significant interest in electronic applications due to simpler device fabrication and better performance. Here we present work on the development of self-assembled fibrous networks of thermotropic triphenylene discotic liquid crystals, where 2,3,6,7,10,11-Hexakishexyloxytriphenylene (HAT6) is studied. The formation of interconnected molecular fibres in acetonitrile-based solvents facilitates thermallyreversible physical-gel (non-covalent) preparation, with the HAT6 network providing mechanical support and containment of the solvent. Furthermore, gel formation is also achieved using an acetonitrile-based iodide/tri-iodide redox liquid electrolyte, and the resulting gel mixture is utilised as an electrolyte in dye-sensitized solar cells (DSSCs). Our results show that it is indeed possible to achieve in situ gel formation in DSSCs, allowing for easy cell fabrication and electrolyte filling. In addition, the gel phase is found to increase device lifetime by limiting solvent evaporation. Differential scanning calorimetry (DSC) and polarising optical microscopy (POM) are used to study gel formation, and it is identified that the thermally reversible gels are stable up to working temperatures of $40{ }^{\circ} \mathrm{C}$. It is found that DSSCs filled with gel electrolyte exhibit longer electron lifetime in the $\mathrm{TiO}_{2}$ photo-anode $(\approx 8.4 \mathrm{~ms}$ in the liquid electrolyte to $\approx 11.4 \mathrm{~ms}$ in the gel electrolytes), most likely due to electron screening from the electrolyte by HAT6. Current-Voltage $(\mathrm{I}-\mathrm{V})$ and electrochemical impedance spectroscopy (EIS) are used to study the effect of gel formation on conductivity and electrochemical properties, and it is found that confinement of the liquid electrolyte into a gel phase does not significantly reduce ionic conductivity, a problem common with solid-state polymer electrolytes. A 3.8 mM HAT6 gel electrolyte DSSC exhibited a PCE of $6.19 \%$ vs. a $5.86 \%$ liquid electrolyte reference. Extended device lifetimes studies showed that the gels increase stability of the DSSCs by reducing the rate of solvent evaporation.
\end{abstract}

\section{Introduction}

Discotic liquid crystals (DLCs) are a class of mesophases formed by disc-shaped molecules[1-3]. These materials are known to form columnar mesophases, where the molecules $\pi$-stack into molecular columns, and in the bulk several columns arrange together either in rectangular, hexagonal, or other packing geometries[4-6]. DLCs are also known to exhibit noncolumnar phases, such as the nematic phase $[7,8]$, and, depending on the interaction between the bulk phase and chemical composition of individual molecules, LC materials can be tailored to diverse applications[9-11]. Examples of optoelectronic applications of DLCs include their use as organic semiconductors in solar cells[12-14], optical compensation layers in display panels[15], and more recently as low-molecular-mass organic gelators (LMOGs)[16-19]. In this paper, we 
explore the formation of physical gels using the symmetric triphenylene DLC 2,3,6,7,10,11Hexakishexyloxytriphenylene (HAT6) (Fig 1.a inset). More specifically, we explore its role as a gelating agent for an acetonitrile-based redox liquid electrolyte, and apply the resulting gel electrolytes to dye sensitized solar cells (DSSCs). Acetonitrile has been the solvent of choice for electrolyte applications in DSSCs, using Iodide/tri-Iodide $\left(\mathrm{I}^{-} / \mathrm{I}_{3}{ }^{-}\right)[20]$ as well as Cobalt (III/II) complex redox electrolytes[21], to fabricate DSSCs with efficiencies up to $13 \%$. Therefore, gel formation in acetonitrile based electrolytes using triphenylene DLC HAT6 is very exciting, and a promising prospect in photovoltaic applications.

There are several ways to describe gel formation depending on the physical mechanism and chemistry of the gelation process. Gelators which are based on the formation of covalent bonds to form a network are called chemical-gelators/gels[22], whereas gelators which are bonded by non-covalent interactions, such as hydrogen bonding or $\pi-\pi$ interactions, are called physical gels. Sometimes more specifically, gelators based on interacting $\pi$-systems are classified as $\pi$ gels[23].

Within the gel, an interconnected network of a gelator macroscopically confines a solvent such that bulk flow is limited. The gels described here are formed by fibres of condensed columns of HAT6 molecules[24], where the molecules $\pi$-stack to form the fibres. Triphenylene derivatives have four flat fused rings that exhibit an extended conjugated pi-electron system (inset in Fig 1.a). Triphenylene gels can be made using both symmetric and asymmetric substitutions in the periphery alkyl chains. Previous studies focusing on the use of triphenylenes in gels have taken two different approaches[16],[25,26].

In the first approach, gelators are added to a liquid crystalline matrix[25,26], which has shown to stabilize the columnar phase, improve columnar packing and increase the hole conductivity in the materials. Similar gelators have also been added to calamitic liquid crystals to modulate their electro-optic properties[27]. An interesting property of external gelators added to LC mesophases is that depending on whether the liquid crystalline transition temperature $\mathrm{T}_{\mathrm{I}-\mathrm{N}}$ is greater or lower than the solution-gelation temperature $\left(T_{\text {sol-gel }}\right)$, we get either a disordered gel $\left(T_{\text {I- }}\right.$ $\left.{ }_{\mathrm{N}}<\mathrm{T}_{\text {sol-gel }}\right)$ or highly ordered $\left(\mathrm{T}_{\text {sol-gel }}<\mathrm{T}_{\mathrm{I}-\mathrm{N}}\right)$ LC templated gel[26]. Several types of gelators have been investigated, including photo-responsive (cis-trans azo-benzenes) and thermo-responsive gelators[26].

In the second approach to LC gel fabrication, which is more relevant to the mechanism of gelation studied in this research, LC molecules are used as the gelators in a solvent medium[16]. The bulk LCs phase-separates from the solvent, hence the choice of solvent is very important in 
the gel formation. In addition to phase separation, intermolecular interactions (e.g. hydrogen bonding and $\pi-\pi$ stacking) lead to the formation of long-range fibrous aggregates that ultimately results in a reversible temperature dependent LC network that supports a solvent. Several approaches have been taken to realize these " $\pi$-organogels" using triphenylene-based molecules[28]. A common feature of these approaches is the use of symmetric and asymmetric substitutions of one of the alkyl chains of a symmetric DLC with a functional group, such as an amide[18], alcohol[29], Imidazole[30] or carboxylic group[16]. From previous studies, it can be noted that the nature of the substituent group has a strong effect on gel formation in particular solvents. For example, in the case of an acidic functional group, gel formation was strongly favoured in alcohol based solvents, particularly methanol[16] due to hydrogen bonding. In almost all studies, gel formation is governed by the interplay of intermolecular interactions and solvation effects of the functional groups [26].

DSSCs are a class of third generation solar cell and are promising candidates for future renewable energy applications[31]. In 1991 O'Regan and Grätzel published their work on the use of mesoporous $\mathrm{TiO}_{2}$ for dye adsorption[20], and the high efficiency combined with easy fabrication make these hybrid organic/inorganic devices very appealing[32]. However, one of the lasting challenges with conventional DSSCs has been the volatile nature of the iodide/tri-iodide redox liquid electrolyte that typically uses acetonitrile as a solvent[33]. Solvent evaporation and leakage have been key factors which limit device lifetime, and there is nearly two decades worth of work on the development of approaches to mitigate these problems[34-37]. These approaches have included the use of solid hole transport layers[38,39], solid polymer[40], quasi-solid gel electrolytes[34,41,42], alternative low vapour pressure solvents[43], and ionic liquids[44].

It is demonstrated that self-assembled physical gels using triphenylene DLCs as gelators can be used to form gel electrolytes that can be applied in DSSCs. Gel formation occurs in-situ, allowing convenient fabrication and complete infiltration of the mesoporous Titanium dioxide $\left(\mathrm{TiO}_{2}\right)$. It is shown that the gels lead to reduced electron recombination at the $\mathrm{TiO}_{2}$ interface. Furthermore, significant enhancement in the device lifetime of the gel electrolyte based cells is measured as compared to standard liquid electrolyte DSSCs, as a result of solvent retention in the gel network.

2. Materials, mixture formulation and fabrication

\subsection{Materials}

2,3,6,7,10,11-Hexakishexyloxytriphenylene (HAT6) (SYNTHON Chemicals) DLC was used as received. Acetonitrile (Sigma Aldrich) was used as a solvent in both pure and electrolyte- 
based gels. Iodide/tri-iodide redox liquid electrolyte was prepared by mixing $0.5 \mathrm{M}$ 4-tertbutylpyridine (Sigma Aldrich), 0.5 M lithium iodide (LiI) (Sigma Aldrich), 0.6 M 1-Methyl-3propylimidazolium iodide (MPII) (Sigma Aldrich), and 0.05M iodine ( $\mathrm{I}_{2}$ ) (Sigma Aldrich) in acetonitrile.

For DSSC photoanodes, a compact layer of $\mathrm{TiO}_{2}$ was prepared by spin-coating a titanium iso-propoxide solution $(35 \mu \mathrm{L} 2 \mathrm{M} \mathrm{HCl}$ (Fisher) and $350 \mu \mathrm{L}$ Titanium Isopropoxide in $5 \mathrm{ml}$ anhydrous ethanol (Sigma Aldrich)). To prepare mesoporous titanium dioxide $\left(\mathrm{TiO}_{2}\right)$ (Dyesol 18 NR-T Transparent Titania paste) was used. Fluorine-doped tin oxide FTO glass (Sigma Aldrich) was used as a substrate for both the anode and cathode. Platinum paste (PT-1 Dyesol) was used a catalyst for electrolyte regeneration.

\subsection{Gel mixture formation}

To prepare HAT6 DLC gels, varying concentrations of HAT6 were added to either pure acetonitrile or liquid electrolyte. This was followed by heating the sample in a vial in an oven at $45^{\circ} \mathrm{C}$ to melt the crystalline (Cr) HAT6 into the solution. The heated sample was then sonicated, followed by heating again in an oven at the same temperature $\left(45^{\circ} \mathrm{C}\right)$. Subsequently, cooling the sample to room temperature results in gel formation (Fig 1.g), and this temperature dependent transition from the gel phase to a solution was process is found to be repeatable.

\subsection{Cell Fabrication}

Symmetric electrode sandwich cells for current-voltage (I-V) measurements were prepared using Indium Tin Oxide (ITO) coated glass. A $25 \mu \mathrm{m}$ sealing and spacer film (Meltonix 1170-25, Solaronix) was used to maintain a fixed gap between the substrates. For $9.7 \mu \mathrm{m}$ ITO-ITO test cells, glass spacers dispersed in UV-curable glue (NOA-68) were used. Subsequently, for linear sweep voltammetry measurements, platinum-coated Fluorine doped Tin Oxide (FTO) substrates were used to make sandwich cells with $60 \mu \mathrm{m}$ spacer film (Meltonix).

For DSSC photo-anode preparation, a $\mathrm{TiO}_{2}$ blocking layer was prepared by spin-coating the compact layer solution at $2000 \mathrm{rpm}$ for $60 \mathrm{~s}$. This was followed by an annealing step at $450^{\circ} \mathrm{C}$ for 30 minutes. Subsequently, mesoporous $\mathrm{TiO}_{2}$ paste was bar-coated using a circular mask (to control the active area across devices) and annealed at $450{ }^{\circ} \mathrm{C}$ to give an $8 \mu \mathrm{m}$ thick mesoporous layer. The substrate was then immersed in a dye solution for 24 hours for dye adsorption. To prepare the counter electrode, Pt paste was spin-coated at $2000 \mathrm{rpm}$ for $30 \mathrm{~s}$, followed by sintering at $450^{\circ} \mathrm{C}$. The cell was then sealed using $25 \mu \mathrm{m}$ Meltonix film, followed by filling with either liquid electrolyte at room temperature, or HAT6-electrolyte gel at $40{ }^{\circ} \mathrm{C}$ (to fill in the isotropic phase). 


\section{Experimental methods}

A polarising optical microscope (POM) (Olympus BX-60) combined with an integrated heating stage and temperature controller (Linkam TMS 94) were used to visualise gel formation inside sandwich cells as a function of temperature. Differential Scanning Calorimetry (DSC) (Mettler Toledo $823^{\mathrm{e}}$ ) was used to measure phase transitions of the gels, using a cooling and heating rate of $5^{\circ} \mathrm{C} / \mathrm{min}$. To perform I-V measurements on ITO-ITO sandwich cells filled with the gels, a Keithley 4200-SCS parameter analyser was used. DSSC devices were tested under simulated AM1.5 standard conditions using a solar simulator (ABET 2000), and a sourcemeter (Keithley 2400) was used to perform voltage sweeps of the cell to measure the dark and AM1.5 light I-V response. Electrochemical impedance spectroscopy (EIS) (Autolab PGSTAT 302N) of the DSSC devices was performed to measure the small signal response at variable frequencies to study electron lifetimes in the $\mathrm{TiO}_{2}$ and changes in charge transfer resistance at the DSSC counter electrode due to gel formation. Linear sweep voltammetry (LSV) measurements are performed using an Autolab electrochemical interface instrument (PGSTAT 302N). Temperature dependent conductivity measurements were performed using a parameter analyzer (6440A, Wayne Kerr) with the sample placed on a hot-stage (Linkam).

\section{Results and discussion}

Experimental characterization of the gel electrolytes, their application in DSSC and subsequent discussion of the results is summarized in this section.

\subsection{Phase transition analysis}

HAT6 (chemical structure illustrated in inset in Fig 1.a) is a thermotropic DLC that forms a columnar mesophase between $68^{\circ} \mathrm{C}$ and $99{ }^{\circ} \mathrm{C}$ on heating, and exhibits a crystalline (Cr) phase below, and an isotropic phase above, the mesophase temperature range. DSC scans of triphenylene HAT6 are shown in Fig 1.a. HAT6 was found to be soluble in aromatic solvents such as toluene and chlorobenzene, and also some non-aromatic solvents such as chloroform. Interestingly however, it was found that if the HAT6-acetonitrile sample is heated in an oven and cooled back to room temperature, then with of HAT6 to acetonitrile in a certain critical concentration, it was also possible to achieve gel formation by cooling the solution from isotropic. In addition, it was found that gel formation occurs in acetonitrile-based liquid electrolyte as well (see Section 2 for chemical composition).

To understand the gel formation process better, we prepared gel mixtures with varying concentration of the gelator HAT6, the results of which are summarised in Table 1. It was identified that no precipitate is observed in the case of $0.0038 \mathrm{M}$ and $0.0078 \mathrm{M} \mathrm{HAT6}$ in liquid 
electrolyte. At concentrations higher than $0.0078 \mathrm{M}$ precipitation occurred (in addition to gelation), and at lower concentrations (0.00195 M HAT6) incomplete gel formation was observed. Based on these results, it is estimated that the critical gelation concentration of HAT6 in liquid electrolyte (i.e. the lowest concentration of the gelator that supports the formation of a stable gel) is in the range of $0.00195 \mathrm{M}$ to $0.0038 \mathrm{M}$. Higher concentrations did not aid further gel formation.

\begin{tabular}{|c|c|c|c|c|c|c|c|c|c|c|}
\hline $\begin{array}{c}\text { Concentration } \\
\text { of HAT6 (M) }\end{array}$ & 1 & 0.5 & 0.25 & 0.125 & 0.063 & 0.031 & 0.0156 & 0.0078 & 0.0038 & 0.00195 \\
\hline $\begin{array}{c}\text { Precipitate/ } \\
\text { Gel/Liquid }\end{array}$ & P/G & P/G & P/G & P/G & P/G & P/G & P/G & G & G & G/L \\
\hline
\end{tabular}

Table 1. Summary of the effect of HAT6 concentration in liquid electrolyte on gel formation. P/G: gel formation with HAT6 precipitation at the bottom of the vial, G: gel formation with no precipitation, and G/L: partial gel formation with co-existing liquid phase.

In order to quantify the phase transition temperatures of the gels, POM and DSC were utilized. Optical textures of a 0.0038 M HAT6-acetonitrile gel in a $9 \mu \mathrm{m}$ ITO-ITO cell (with planar alignment layers) at various temperatures are illustrated in Fig 1.(c-f). The cell was initially heated to $40{ }^{\circ} \mathrm{C}$ and capillary filled in the isotropic phase (Fig 1.c). Subsequently, the cell was slowly cooled and it was found that complete gel formation occurred at a temperature of $29{ }^{\circ} \mathrm{C}$ (Fig 1.d). This is characterised by the formation of birefringent HAT6 fibres that seem to branch out and interlink in places, and the individual width of such fibres is of the order of a several microns. On heating, the gel phase was found to be stable up to a temperature of $36^{\circ} \mathrm{C}$, where the gel started to break, and further heating up to $42{ }^{\circ} \mathrm{C}$ resulted in a complete isotropic phase (Fig 1.f). It can be seen in Fig 1.d that the width of individual fibres (measured to be $\approx 3 \mu \mathrm{m}$ ) is nearly three orders of magnitude larger than the width of each HAT6 molecular column $(\approx 2.5 \mathrm{~nm})$, where the diameter of individual molecular columns is dependent on the size of individual HAT6 molecules[45], so it is hypothesised that the self-assembled fibres visible in the POM must be bundles of molecular columns. Previous studies on amide and carboxylic substituted triphenylenes reported fibre widths of 90-180 nm, that were also considered to be bundles of individual columns[16].

DSC analysis of the gels (0.0038 M HAT6 in liquid electrolyte) was also performed (Fig 1.b). It is interesting to note that during both heating and cooling, very broad transitions were measured, with a width of almost $3{ }^{\circ} \mathrm{C}$ on cooling. On heating, it was observed that in addition to a broad transition that starts at $26^{\circ} \mathrm{C}$, several smaller transitions were also observed (red circles in Fig 1.b, black line). It is clear that there are no sharp thermodynamic transitions as is the case with pure materials (e.g. the transition of HAT6 in Fig 1.a), and instead the transitions are distributed 
with temperature. It is also interesting to note that while HAT6 has a Cr-LC transition at an elevated temperature of $68^{\circ} \mathrm{C}$, in the gels we get a complete clearing (i.e. an isotropic phase) at $\approx$ $40{ }^{\circ} \mathrm{C}$ due to solvent mediated interactions.

Gel formation of HAT6 in acetonitrile and $\mathrm{I}^{-} / \mathrm{I}_{3}{ }^{-}$liquid electrolyte inside glass bottles on a bench top is shown in Fig 1.g and Fig 1.h, respectively. The gels were heated to isotropic inside an oven, and left to cool passively to room temperature. Cloudy precipitates (turbid) are observed to initially form at the bottom of the sample bottles in Fig 1.g.ii, corresponding to the formation of DLC fibres. Further cooling (Fig 1.g.iii) leads to the elongation and cross-linking of the fibres, leading to the formation of a complete gel. Inversion of the sample bottles with the flow of liquid (Fig 1.g.iv) is indicative of gel formation.

\subsection{Conductivity and ionic diffusion studies}

Conductivity is dominated by the ionic diffusion of lithium cations $\left(\mathrm{Li}^{+}\right)$and iodine anions $\left(\mathrm{I}^{-} / \mathrm{I}_{3}{ }^{-}\right)$in the electrolyte medium. The addition of gelators and other non-conducting additives to the electrolyte typically results in an increase in transport resistance because of a reduction in diffusion coefficient of the ions[46] Conductivity measurements on HAT6-based gel electrolytes in symmetric ITO-ITO cells are presented in Fig 2.(a, b). The measurements here correspond to non-steady state conditions (i.e. fast sweeps, $\approx 70 \mathrm{mV} / \mathrm{s}$ ) with ITO electrodes that do not catalyse the $\mathrm{I}^{-} / \mathrm{I}^{3-}$ redox reaction. A liquid electrolyte was used as a reference and from Fig 2 it is evident that the conductivity of the gel and liquid electrolytes were measured to be very similar: at an applied voltage of $2 \mathrm{~V}$ to a $25 \mu \mathrm{m}$ cell, the liquid electrolyte average conductivity is estimated to be $(1.33 \pm 0.21) \times 10^{-3} \mathrm{~S} / \mathrm{m}$, whereas the $0.0038 \mathrm{M}$ HAT6 gel electrolyte conductivity is estimated as $(1.28 \pm 0.16) \times 10^{-3} \mathrm{~S} / \mathrm{m}$, both using a simple linear resistor model. There is some sample-to-sample variation of conductivity, which is likely the result of experimental and cell fabrication variations. The data indicates no significant decrease in conductivity upon gelation. Based on the conductivity results, it is hypothesized that HAT6 does not directly contribute towards conductivity through chemical reactions with the electrolyte, and the reactions governing conduction are:

$$
\begin{aligned}
& \mathrm{I}_{2}+\mathrm{I}^{-} \rightarrow \mathrm{I}^{-} \\
& \mathrm{I}^{-}+2 \mathrm{e}^{-} \rightarrow 3 \mathrm{I}^{-}
\end{aligned}
$$

An ITO-ITO test cell filled with 0.0038 M gel electrolyte (Cell 3 from Fig 2.a) was analysed under reflection using POM. Optical micrographs under crossed polarizers are shown before (Fig 2.c) and after (Fig 2.d) a $45^{\circ}$ anti-clockwise rotation. It is interesting to note the occurrence of birefringent fringes on the fibers, and that the sequence of bright and dark fringes changes under rotation (see enlarged regions compared after compensating for rotation). This is possible evidence 
towards the twisting of molecular column bundles inside the fibres, resulting in regions of low and high effective birefringence under crossed polarizers that vary under rotation.

In order to quantify diffusion characteristics of the tri-iodide ionic species $\mathrm{I}_{3}^{-}$(which has the limiting concentration) in the liquid and gel electrolytes, steady state linear sweep voltammetry measurements were performed in symmetric sandwich cells. The results are summarised in Fig 3.a, which also shows the calculated diffusion coefficients by using limiting current values [46,47],using the equation:

$$
J_{\text {lim }}=\frac{2 n F c_{o} D\left[I_{3}^{-}\right]}{d}
$$

where $n$ is taken to be two (number of electrons needed to reduce $\mathrm{I}_{3}^{-}$to $\mathrm{I}^{-}$), $d$ is the cellgap, $F$ is the faraday constant, $\mathrm{c}_{0}$ is the concentration of the $\mathrm{I}_{3}{ }^{-}[46]$, and $D\left[I_{3}{ }^{3}\right.$ is the tri-iodide diffusion coefficient. It can be seen that the diffusion coefficient is slightly higher in the liquid electrolyte $(1.46 \pm 0.24) \times 10^{-7} \mathrm{~cm}^{2} \cdot \mathrm{s}^{-1}$ than the $0.0038 \mathrm{M}$ gel electrolyte $(1.04 \pm 0.11) \times 10^{-7} \mathrm{~cm}^{2} \cdot \mathrm{s}^{-1}$. This is in agreement with our EIS measurements (Fig 4.c), where it was found that the gel electrolytes exhibit a higher charge transfer resistance at the platinum cathode. Previous studies of ionic diffusion in polymer gel electrolytes report a decrease in ionic conductivity/diffusion coefficient with gel formation [46,48]. However, other studies utilising gel electrolytes instead report no significant change in conductivity on gel formation, as the ionic species are free to move within a continuously liquid electrolyte that is constrained within a porous insulating gel network[41].

The variation of the conductivity of gel and liquid electrolytes as a function of temperature is shown in Fig 3.b. The measurements were performed on using an LCR bridge component analyzer (Wayne Kerr), at an applied voltage of $1 \mathrm{~V}$ at $1 \mathrm{KHz}$. The samples were placed on a hotstage and conductivity as a function of temperature was recorded. The temperature dependent conductivity behaviour of most common liquid electrolytes follows the Arrhenius relation[49], where a straight line relationship exists between the natural log of the rate constant (in this case conductivity) against inverse temperature (1/T). In Fig 3.b it can be assumed that the $\ln (\sigma)$ vs. 1/T plot is a straight line for the liquid electrolyte, despite the slight curve at high temperatures (most likely due to solvent evaporation when testing), but the overall trend can be assumed to be somewhat linearly increasing. However, in the case of the gel electrolyte, it can be seen that the $\ln (\sigma)$ vs. $1 /$ T curve does not follow the Arrhenius straight-line relation at all, and there is significant temperature dependence and resultant deviation. It can be seen that there is a stationary point in the curve at $45^{\circ} \mathrm{C}$, close to the clearing of the gel state to an isotropic liquid. The overall trend is an increase in conductivity as a result of increasing temperature, however it is clear by comparing 
the gradients of the liquid electrolyte and gel electrolyte curves at lower temperatures, that during the gel phase the incremental increase in conductivity of the gel electrolyte is less than the liquid electrolyte, followed by a significant change (and subsequent increase at higher temperature) in this gradient beyond the gel clearing temperature. Therefore, analysing the steady-state LSV and temperature dependent conductivity measurements, it is clear that there is interaction between the HAT6 fibers and diffusing ionic species which results in the lowering of the diffusion constant slightly. This could either be a manifestation of the trapping of $\mathrm{I}_{3}^{-}$ions due to the DLC fibers, or another possibility is that lowering of the diffusion constant is a result of the partial coverage the ITO electrodes by insulating HAT6. In the next section, we apply the redox gel electrolytes to DSSCs to analyze the effects of gel formation on photovoltaic parameters.

Since no DLC precipitation inside the bottle was observed in the case of $0.0038 \mathrm{M}$ and 0.0078M HAT6 in liquid electrolyte, these two concentrations were used to prepare solar cells. A schematic of the devices is shown in Fig 4.f. Cells were prepared using the standard procedure (see Methods), with the exception that the cells were filled at an elevated temperature of $40{ }^{\circ} \mathrm{C}$. This temperature is high enough to melt the gel into an isotropic liquid, allowing complete filling, but not so high that the acetonitrile/TBP components of the electrolyte evaporate. Photovoltaic cells containing each gel type were prepared, and standard iodide/tri-iodide redox liquid electrolyte filled devices were used as a reference in the experiment. I-V curves corresponding to the highest performing devices of each category tested are shown in Fig 4.b. The dark I-V curves of the solar cells (Fig 4.a) are found to be very similar. It can be observed from the inset in Fig 4.b that the highest photovoltaic efficiency was measured in a cell utilising the $0.0038 \mathrm{M}$ gel electrolyte. The open circuit voltage $\left(\mathrm{V}_{\mathrm{OC}}\right)$ and fill factor $(\mathrm{FF})$ are relatively similar across the devices, and any differences between devices may be attributed to device characteristics rather than the electrolyte. The highest performing devices in all cases are distinguished by high short-circuit currents (Jsc), and the highest $\mathrm{J}_{\mathrm{SC}}\left(13.3 \mathrm{~mA} / \mathrm{cm}^{2}\right)$ was measured in a $0.0038 \mathrm{M}$ gel device.

\subsection{Electrochemical Impedance spectroscopy}

Electrochemical impedance spectroscopy (EIS) is performed to study the various interfaces involved in the DSSC devices [50]. Typical EIS spectra yield information about different intra-device processes depending on the magnitude of applied bias, illumination level and frequency of the small signal applied [51]. Fig 4.(c, d) show the EIS results for the case of a $-0.7 \mathrm{~V}$ forward bias under dark conditions. Significant research has been performed to identify the frequency dependence of various interfaces in DSSC devices[51]. It is generally accepted that the high frequency semicircle corresponds to processes at the Pt counter electrode, the middle semicircle is characteristic of impedances corresponding to electrons injected into the $\mathrm{TiO}_{2}$ anode, and 
the final (low frequency) region corresponding to the diffusion of the iodide ions in the electrolyte [52]'[51]. It can be seen from Fig 4.c that the charge transfer impedance at the Pt electrode (the width of the first semi-circle) is $\approx 25 \Omega$ in the case of a 0.0038 M HAT6 gel electrolyte, which is higher than the $\approx 7 \Omega$ measured in the case of the liquid electrolyte. It is hypothesized that this is due to the presence of HAT6 fibres that partially cover the counter-electrode, thereby reducing the effective catalytic area for tri-iodide reduction, combined with a slight reduction in ionic diffusion rate due to gel formation.

Furthermore, using the middle frequency peak positions $(10-100 \mathrm{~Hz})$ in Fig 4.d, it can be seen that there is a left-shift in the peak position for the gel electrolyte. This may be interpreted as an increase in the effective lifetime of the injected carriers in the $\mathrm{TiO}_{2}$ anode. These lifetimes are calculated to be $\approx 8.4 \mathrm{~ms}$ in the case of the reference liquid electrolyte and $11.4 \mathrm{~ms}$ in the case of the gel electrolyte. It is hypothesised that this increase in electron lifetime is due to the screening effect of the insulating $\mathrm{HAT} 6$ at the $\mathrm{TiO}_{2}$-electrolyte interface, which helps reduce recombination between the mesoporous $\mathrm{TiO}_{2}$ and the $\mathrm{I}_{3}{ }^{-}$ions. Electron screening takes place due to the HAT6 fibers in the electrolyte, as the fiber network reduces the extent of the direct contact between photo-injected the electrons in the $\mathrm{TiO}_{2}$ conduction band and the $\mathrm{I}_{3}{ }^{-}$ions in the electrolyte, effectively passivating the $\mathrm{TiO}_{2}$ to an extent, leading towards a reduction in the back transfer of electrons. A similar effect was found in a previous study by Rui-Xuan et.al [42]. The overall efficiency of the gel electrolytes DSSCs in our experiment (inset Fig 4.b) was found to be similar (and in some cases greater) than the liquid electrolyte devices. It is speculated that the increase in charge transfer resistance at the Pt counter electrode (which reduced the short circuit current Jsc) is compensated by an increase in electron lifetime in the $\mathrm{TiO}_{2}$, keeping device efficiencies almost constant for both reference and gel electrolyte devices.

\subsection{DLC physical-gel electrolyte based DSSCs}

As discussed earlier, one of the main challenges associated with the widespread commercial adoption of DSSCs is the volatile and corrosive nature of the liquid electrolyte[38], whereby acetonitrile evaporation inevitably degrades device performance. By forming a physical-gel inside a DSSC through the use of a HAT6 liquid electrolyte, it should be possible to increase the lifetime of the photovoltaic devices. With this in mind, lifetime studies were performed over several weeks, and the cells were tested intermittently to evaluate performance over time. Once again, liquid electrolyte cells were used as a reference. It should be noted that DSSC preparation techniques and lifetimes may vary significantly between laboratories, and that what is important is therefore the relative lifetime of the devices. It is expected that industrial sealing and manufacturing techniques would greatly enhance the device lifetimes presented here. 
Fig 5.(a, b) shows the progression of solar cell efficiency as a function of time. The variation of efficiency in the case of 0.0038 M HAT6 gel electrolyte is presented in Fig 5.a, and the corresponding data for reference electrolyte devices is summarized in Fig 5.b. In both cases it is clear that there is a loss in device efficiency over time. However, the degradation is much faster in the reference electrolyte devices, where only one out of four devices (Cell 4, Fig 5.b) had a power conversion efficiency (PCE) of 4\% seven days after fabrication; the other three cells degraded completely within the first week. In the case of the gel electrolytes (Fig 5.a), while there is still continuous degradation with time, the devices are much more stable. We hypothesize that the formation of a gel reduces the rate of solvent evaporation, thereby increasing the device lifetime. In particular, we measured a "levelling off" of the efficiency in the case of Cell 1 and Cell 2 (Fig 5.a) at efficiencies of $\approx 1 \%$ and $2.1 \%$ respectively more than a month after fabrication. All devices tested were stored in opaque sample boxes (unsealed) at room temperature in ambient atmospheric conditions.

Variation of individual performance parameters (Jsc, Voc, fill factor, and device efficiency) as a function of time for a gel-electrolyte DSSC (cell 2 from Fig. 4.a) are summarized in Fig 5.(c, d). The Fill factor of the device remained relatively unchanged over the several weeks that the measurements were performed. However, as discussed earlier, there is decrease in overall device performance efficiency with time. This is best captured by analysing the variation of short circuit current with time, which follows closely the efficiency curve. It is also noteworthy that the open circuit voltage was invariant and did not have a significant impact on device efficiency or degradation. If anything, the open circuit voltage was found to increase from the initial measured value. It is therefore clear that it is the short circuit current which dominates device performance, and keeping it constant is key to maximising lifetime. Optical micrographs under reflection of the active area of the 0.0038 M HAT6 cell (tested in Fig 5.a) illustrate the gel electrolyte on Day 1 (Fig 5.e) and on Day 44 (Fig 5.f). The invariance of the gel texture over a relatively extended period of time is encouraging. This also implies that even after exposing the cell numerous times to 1 sun illumination in a solar simulator, the gel texture is maintained and a gel-sol transition did not occur.

\section{Conclusion}

It has been shown that the symmetric triphenylene molecule HAT6, which forms a hexagonal columnar mesophase, self-assembles into a fibrous network that forms physical-gels in an acetonitrile environment in its pure and liquid electrolyte forms. The gel formation is hypothesized to be a manifestation of the combined effects of intermolecular pi-stacking effects and interactions of the HAT6 alkyl chains with acetonitrile. The critical gelation concentration for 
gelation to take place in acetonitrile based I-/I3- redox electrolyte was identified to a HAT6 concentration in the range of $1.95 \mathrm{mM}$ to $3.8 \mathrm{mM}$. POM analysis of the birefringent fibres showed that the fibers have a typical diameter of $\approx 3 \mu \mathrm{m}$, implying that the fibres are most likely bundles of individual HAT6 molecular columns wound together. Electrical conductivity measurements showed that the measured conductivity was found to be similar in the gel and liquid electrolytes. DSSCs were prepared using the DLC gel electrolytes and it was found that the photovoltaic performance parameters of the gel electrolyte devices were similar to the liquid electrolyte references, with the 0.0038 M HAT6 showing slightly higher short circuit current in some devices. EIS studies showed the presence of higher charge transfer resistance at the counter electrode in the gel devices, complemented with higher electron lifetime due to reduced back electron transfer, and hence lower loss, in the TiO2 anode. Extended device lifetime studies showed that under identical testing, sealing and storage conditions, the gel electrolyte cells exhibited significantly longer lifetimes than reference liquid electrolyte devices. In addition to their use as gel electrolytes in DSSCs, HAT6-based gels can potentially be used for other applications, such as supercapacitors, and it is anticipated that future work on DLC based gelators will become very relevant to such commercial applications.

\section{Acknowledgements}

The authors would like to thank Organica and Dyesol for providing the materials used in this research. We would like to thank Dona T. Galhena for her help in conducting electrochemical impedance spectroscopy and linear sweep voltammetry experiments. In addition, we are grateful to Prof. Gehan Amaratunga for allowing us to use his lab equipment to perform those measurements. A.A.K would like to thank the Cambridge Commonwealth European and International Trust (CCEIT) and the Higher Education Commission of Pakistan (HEC) for financial support. Furthermore, we would like to thank James Dolan and Dona T. Galhena for proof-reading the manuscript.

\section{References}

[1] K. Ohta, K. Hatsusaka, M. Sugibayashi, M. Ariyoshi, K. Ban, F. Maeda, R. Naito, K. Nishizawa, A.M. Van de Craats, J.M. Warman, Discotic Liquid Crystalline Semiconductors, Mol. Cryst. Liq. Cryst. 397 (2003) 25-45. doi:10.1080/714965592.

[2] R.J. Bushby, O.R. Lozman, Discotic liquid crystals 25 years on, Curr. Opin. Colloid Interface Sci. 7 (2002) 343-354. doi:10.1016/S1359-0294(02)00085-7.

[3] S. Sergeyev, W. Pisula, Y.H. Geerts, Discotic liquid crystals: a new generation of organic semiconductors., Chem. Soc. Rev. 36 (2007) 1902-29. doi:10.1039/b417320c.

[4] E. Pouzet, V. De Cupere, C. Heintz, J.W. Andreasen, D.W. Breiby, M.M. Nielsen, P. Viville, R. Lazzaroni, G. Gbabode, Y.H. Geerts, Homeotropic alignment of a discotic liquid crystal induced by a sacrificial layer, J. Phys. Chem. C. 113 (2009) 14398-14406. doi:10.1021/jp9035343.

[5] S. Kumar, Discotic liquid crystal-nanoparticle hybrid systems, NPG Asia Mater. 6 (2014) e82. 
doi:10.1038/am.2013.75.

[6] Y. Bouligand, Defects and textures of hexagonal discotics, J. Phys. 41 (1980) 1307-1315. doi:10.1051/jphys:0198000410110130700.

[7] H.K. Bisoyi, S. Kumar, Discotic nematic liquid crystals: science and technology., Chem. Soc. Rev. 39 (2010) 264-85. doi:10.1039/b901792p.

[8] B.R. Kaafarani, Discotic Liquid Crystals for Opto-Electronic Applications † †, Chem. Mater. 23 (2011) 378396. doi:10.1021/cm102117c.

[9] D. Högberg, B. Soberats, S. Uchida, M. Yoshio, L. Kloo, H. Segawa, T. Kato, Nanostructured TwoComponent Liquid-Crystalline Electrolytes for High-Temperature Dye-Sensitized Solar Cells, Chem. Mater. 26 (2014) 6496-6502. doi:dx.doi.org/10.1021/cm503090z.

[10] J. Sakuda, E. Hosono, M. Yoshio, T. Ichikawa, T. Matsumoto, H. Ohno, H. Zhou, T. Kato, Liquid-crystalline electrolytes for lithium-ion batteries: Ordered assemblies of a mesogen-containing carbonate and a lithium salt, Adv. Funct. Mater. 25 (2015) 1206-1212. doi:10.1002/adfm.201402509.

[11] S.M. Said, S.R. Sahamir, M.F.M. Sabri, M.A. Kamarudin, K. Hayashi, A.Z.S. Zulkifli, T. Nakajo, M. Kubouchi, Y. Miyazaki, Polymer electrolyte liquid crystal mixtures as phase-dependent thermoelectric materials, Mol. Cryst. Liq. Cryst. 642 (2017) 9-17. doi:10.1080/15421406.2016.1262668.

[12] L. Li, S. Kang, J. Harden, Q. Sun, X. Zhou, L. Dai, A. Jakli, S. Kumar, Q. Li, Nature inspired light harvesting liquid crystalline porphyrins for organic photovoltaics, Liq. Cryst. 35 (2008) 233-239. doi:10.1080/02678290701806584.

[13] Y.S. Cheng, C. Gau, Efficiency improvement of organic solar cells with imprint of nanostructures by capillary force lithography, Sol. Energy Mater. Sol. Cells. 120 (2014) 566-571. doi:10.1016/j.solmat.2013.09.040.

[14] L. Schmidt-Mende, a Fechtenkötter, K. Müllen, E. Moons, R.H. Friend, J.D. MacKenzie, Self-organized discotic liquid crystals for high-efficiency organic photovoltaics., Science. 293 (2001) 1119-22. doi:10.1126/science.293.5532.1119.

[15] H. Search, C. Journals, A. Contact, M. Iopscience, I.P. Address, Performance of a Novel Optical Compensation Film Based on Negative Discotic Compound for Wide-Viewing-Angle Birefringence of Twisted-Nematic Liquid-Crystal Displays, 143 (n.d.).

[16] I. Muñoz Resta, V. Manzano, F. Cecchi, C. Spagnuolo, F. Cukiernik, P. Di Chenna, Supramolecular Assembly of $\mathrm{pH}$-Sensitive Triphenylene Derived $\pi$-Gelators and Their Application as Molecular Template for the Preparation of Silica Nanotubes, Gels. 2 (2016) 7. doi:10.3390/gels2010007.

[17] P. Terech, R.G. Weiss, Low Molecular Mass Gelators of Organic Liquids and the Properties of Their Gels., Chem. Rev. 97 (1997) 3133-3160. doi:10.1021/cr9700282.

[18] Y.F. Bai, K.Q. Zhao, P. Hu, B.Q. Wang, Y. Shimizu, Synthesis of Amide Group Containing Triphenylene Derivatives as Discotic Liquid Crystals and Organic Gelators, Mol. Cryst. Liq. Cryst. 509 (2009) 60/[802]76/[818]. doi:10.1080/15421400903112069.

[19] D.J. Abdallah, R.G. Weiss, Organogels and low molecular mass organic gelators, Adv. Mater. 12 (2000) 12371247. doi:10.1002/1521-4095(200009)12:17<1237::AID-ADMA1237>3.0.CO;2-B.

[20] M. Gratzel, B. O'Regan, A low-cost, high-efficiency solar cell based on dye-sensitised colloidal TiO2 films, Nature. 354 (1991) 56-58. doi:10.1038/353737a0.

[21] S. Mathew, A. Yella, P. Gao, R. Humphry-Baker, B.F.E. Curchod, N. Ashari-Astani, I. Tavernelli, U. Rothlisberger, M.K. Nazeeruddin, M. Grätzel, Dye-sensitized solar cells with 13\% efficiency achieved through the molecular engineering of porphyrin sensitizers, Nat. Chem. 6 (2014) 242-247. doi:10.1038/nchem.1861.

[22] G. Deng, C. Tang, F. Li, H. Jiang, Y. Chen, Covalent cross-linked polymer gels with reversible sol-gel transition and self-healing properties, Macromolecules. 43 (2010) 1191-1194. doi:10.1021/ma9022197.

[23] S.S. Babu, V.K. Praveen, A. Ajayaghosh, Functional pi-gelators and their applications, Chem. Rev. 114 (2014) 1973-2129. doi:10.1021/cr400195e.

[24] C. DESTRADE, M.C. MONDON, J. MALTHETE, Hexasubstituted Triphenylenes : a New Mesomorphic Order, Le J. Phys. Colloq. 40 (1979) C3-17-C3-21. doi:10.1051/jphyscol:1979305.

[25] N. Mizoshita, H. Monobe, M. Inoue, M. Ukon, T. Watanabe, Y. Shimizu, K. Hanabusa, T. Kato, The positive effect on hole transport behaviour in anisotropic gels consisting of discotic liquid crystals and hydrogenbonded fibres., Chem. Commun. (Camb). 1 (2002) 428-9. http://www.ncbi.nlm.nih.gov/pubmed/12120525.

[26] T. Kato, Y. Hirai, S. Nakaso, M. Moriyama, Liquid-crystalline physical gels, Chem. Soc. Rev. 36 (2007) 18571867. doi:10.1039/b612546h.

[27] N. Mizoshita, Y. Suzuki, K. Kishimoto, K. Hanabusa, T. Kato, Electrooptical properties of liquid-crystalline physical gels: a new oligo(amino acid) gelator for light scattering display materialsElectronic supplementary information (ESI) available: AFM and SEM images of the aggregates of gelators 1???3. See http://w, J. Mater. Chem. 12 (2002) 2197-2201. doi:10.1039/b201484j.

[28] A. Kotlewski, Highly Mobile Triphenylene Based Organogel Networks, 2009.

[29] A. Kotlewski, B. Norder, W.F. Jager, S.J. Picken, E. Mendes, Can morphological transitions in fibrils drive stiffness of gels formed by discotic liquid crystal organogelators?, Soft Matter. 5 (2009) 4905-4913. doi:10.1039/B909622A. 
[30] M. Kimura, T. Hatanaka, H. Nomoto, J. Takizawa, T. Fukawa, Y. Tatewaki, H. Shirai, Self-assembled helical nanofibers made of achiral molecular disks having molecular adapter, Chem. Mater. 22 (2010) 5732-5738. doi:10.1021/cm102276a.

[31] B.E. Hardin, H.J. Snaith, M.D. McGehee, The renaissance of dye-sensitized solar cells, Nat. Photonics. 6 (2012) 162-169. doi:10.1038/nphoton.2012.22.

[32] M. Grätzel, Dye-sensitized solar cells, J. Photochem. Photobiol. C Photochem. Rev. 4 (2003) 145-153. doi:10.1016/S1389-5567(03)00026-1.

[33] J. Wu, Z. Lan, J. Lin, M. Huang, Y. Huang, L. Fan, G. Luo, Electrolytes in dye-sensitized solar cells, Chem. Rev. 115 (2015) 2136-2173. doi:10.1021/cr400675m.

[34] P. Wang, S.M. Zakeeruddin, J.E. Moser, M.K. Nazeeruddin, T. Sekiguchi, M. Grätzel, A stable quasi-solidstate dye-sensitized solar cell with an amphiphilic ruthenium sensitizer and polymer gel electrolyte., Nat. Mater. 2 (2003) 402-7. doi:10.1038/nmat904.

[35] F. Cao, G. Oskam, P.C. Searson, A Solid State, Dye Sensitized Photoelectrochemical Cell, J. Phys. Chem. 99 (1995) 17071-17073. doi:10.1021/j100047a003.

[36] H.M. Upadhyaya, S. Senthilarasu, M.H. Hsu, D.K. Kumar, Recent progress and the status of dye-sensitised solar cell (DSSC) technology with state-of-the-art conversion efficiencies, Sol. Energy Mater. Sol. Cells. 119 (2013) 291-295. doi:10.1016/j.solmat.2013.08.031.

[37] P. Wang, S.M. Zakeeruddin, M. Graetzel, High efficiency dye-sensitized nanocrystalline solar cells based on ionic liquid polymer gel electrolyte, Chem. Commun. 5 (2002) 2972-2973.

[38] B. Li, L. Wang, B. Kang, P. Wang, Y. Qiu, Review of recent progress in solid-state dye-sensitized solar cells, Sol. Energy Mater. Sol. Cells. 90 (2006) 549-573. doi:10.1016/j.solmat.2005.04.039.

[39] T. Leijtens, I.-K. Ding, T. Giovenzana, J.T. Bloking, M.D. McGehee, A. Sellinger, Hole transport materials with low glass transition temperatures and high solubility for application in solid-state dye-sensitized solar cells., ACS Nano. 6 (2012) 1455-62. doi:10.1021/nn204296b.

[40] T. Asano, S. Uchida, T. Kubo, Y. Nishikitani, Dye-Sensitized Solar Cells Fabricated with Novel Polymeric Solid Electrolyte Films, Energy Convers. (2003) 212-215.

[41] P. Wang, S.M. Zakeeruddin, P. Comte, I. Exnar, M. Grätzel, Gelation of ionic liquid-based electrolytes with silica nanoparticles for quasi-solid-state dye-sensitized solar cells, J. Am. Chem. Soc. 125 (2003) 1166-1167. doi:10.1021/ja029294+.

[42] R.X. Dong, S.Y. Shen, H.W. Chen, C.C. Wang, P.T. Shih, C.T. Liu, R. Vittal, J.J. Lin, K.C. Ho, A novel polymer gel electrolyte for highly efficient dye-sensitized solar cells, J. Mater. Chem. A. 1 (2013) 8471-8478. doi:Doi 10.1039/C3ta11331k.

[43] F. Bella, C. Gerbaldi, C. Barolo, M. Grätzel, Aqueous dye-sensitized solar cells, Chem. Soc. Rev. 44 (2015) 3431-3473. doi:10.1039/C4CS00456F.

[44] R. Kawano, H. Matsui, C. Matsuyama, A. Sato, M.A.B.H. Susan, N. Tanabe, M. Watanabe, High performance dye-sensitized solar cells using ionic liquids as their electrolytes, J. Photochem. Photobiol. A Chem. 164 (2004) 87-92. doi:10.1016/j.jphotochem.2003.12.019.

[45] A.A. Khan, G. Rughoobur, M.A. Kamarudin, A. Sepe, J.A. Dolan, A.J. Flewitt, M.M. Qasim, T.D. Wilkinson, Homologous binary mixtures and improved hole conduction of self-assembled discotic liquid crystals, Org. Electron. 36 (2016) 35-44. doi:10.1016/j.orgel.2016.05.027.

[46] A.G. Kontos, M. Fardis, M.I. Prodromidis, T. Stergiopoulos, E. Chatzivasiloglou, G. Papavassiliou, P. Falaras, Morphology, ionic diffusion and applicability of novel polymer gel electrolytes with LiI/I2., Phys. Chem. Chem. Phys. 8 (2006) 767-76. doi:10.1039/b515113a.

[47] M. Zistler, C. Schreiner, P. Wachter, P. Wasserscheid, D. Gerhard, H.J. Gores, Electrochemical characterization of 1-ethyl-3-methylimidazolium thiocyanate and measurement of triiodide diffusion coefficients in blends of two ionic liquids, Int. J. Electrochem. Sci. 3 (2008) 236-245.

[48] S. Murai, S. Mikoshiba, H. Sumino, T. Kato, S. Hayase, Quasi-solid dye sensitised solar cells filled with phaseseparated chemically cross-linked ionic gels, Chem. Commun. 9 (2003) 1534-1535. doi:10.1039/b302793g.

[49] S. Çavuş, E. Durgun, Poly ( vinyl alcohol ) Based Polymer Gel Electrolytes: Investigation on Their Conductivity and Characterization, 129 (2016) 621-624. doi:10.12693/APhysPolA.129.621.

[50] Q. Wang, J.-E. Moser, M. Grätzel, Electrochemical impedance spectroscopic analysis of dye-sensitized solar cells, J. Phys. Chem. B. 109 (2005) 14945-14953.

[51] J. Bisquert, F. Fabregat-Santiago, Impedance spectroscopy: a general introduction and application to dyesensitized solar cells, Dye. Sol. Cells. (2010) 604.

[52] R. Kern, R. Sastrawan, J. Ferber, R. Stangl, J. Luther, Modeling and interpretation of electrical impedance spectra of dye solar cells operated under open- circuit conditions, Electrochim. Acta. 47 (2002) 4213-4225. 


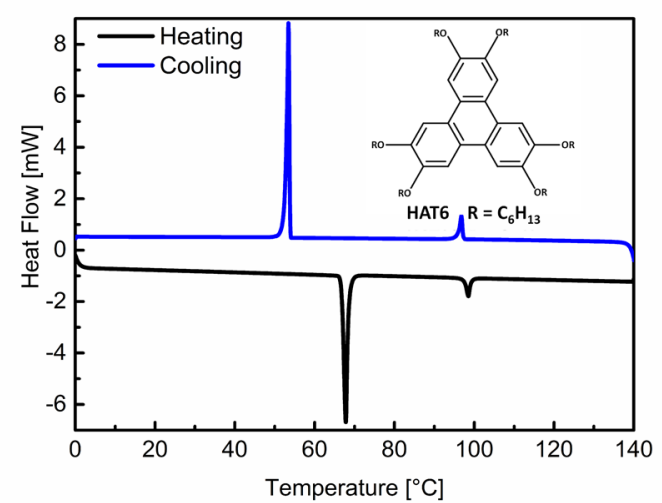

(a)

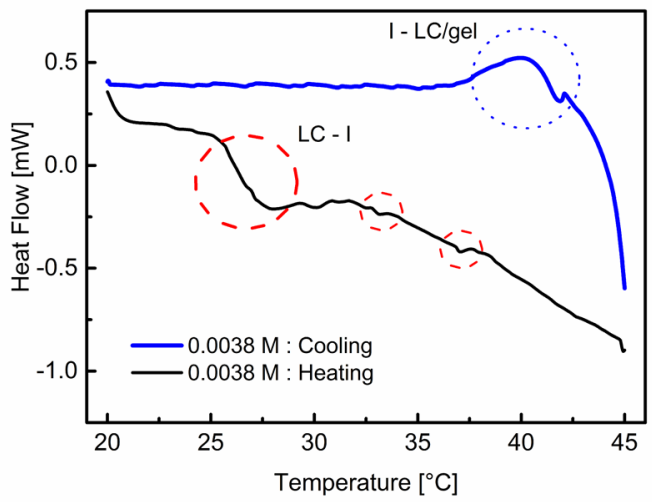

(b)

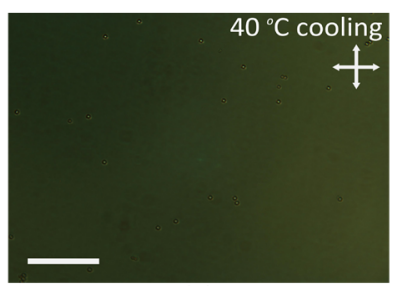

(c)

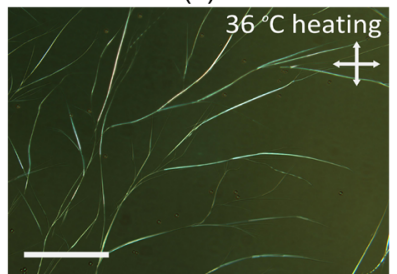

(e)

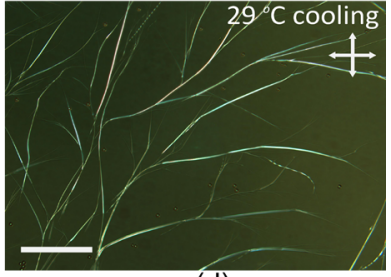

(d)

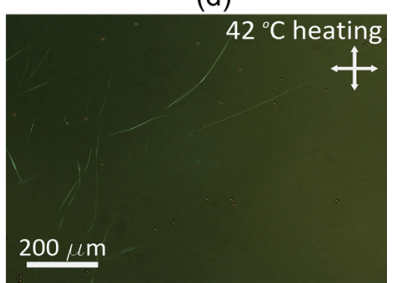

(f)

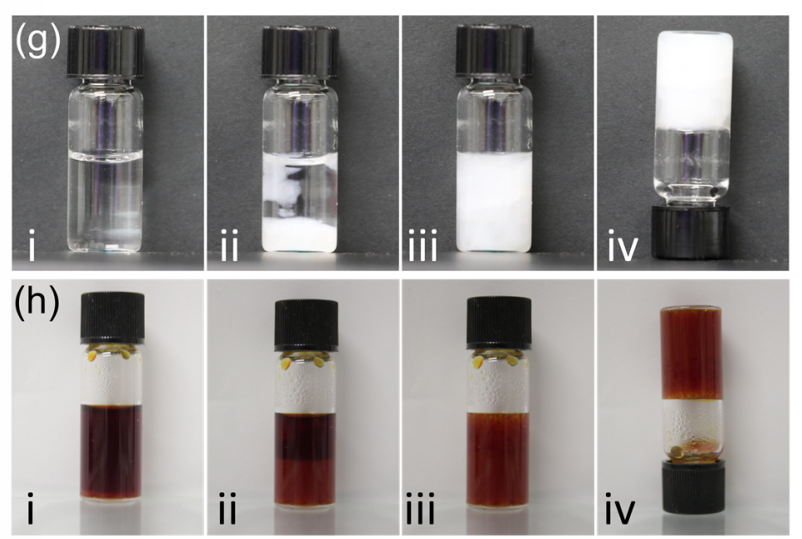

Figure 1. Phase transition measurements. DSC scans of (a) HAT6 and (b) 0.0036 M HAT6 gel electrolyte. Measurements were performed at a heating and cooling rate of $5{ }^{\circ} \mathrm{C} / \mathrm{min}$. Inset in (a) shows chemical structure of HAT6. (c-f) Temperature dependent POM of a $9 \mu \mathrm{m}$ ITO-ITO cell filled with $3.8 \mathrm{mM}$ HAT6 in acetonitrile. The cell is cooled from isotropic (c), to form a gel (d), followed by heating (e) till the birefringent fibres disappear (f). Cooling of $3.8 \mathrm{mM}$ of HAT6 in (g) acetonitrile, and $(\mathrm{h}) \mathrm{I}-/ \mathrm{I}_{3}-$ redox liquid electrolyte mixtures from isotropic (i) to an intermediate state (ii) and eventually forming a gel (iii), that is stable on inversion of the sample vial (iv). The scale bar in (c) - (f) is $200 \mu \mathrm{m}$. 


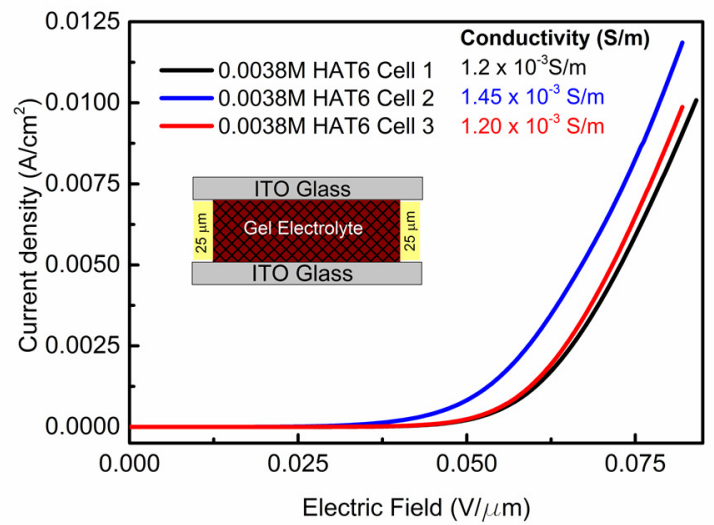

(a)

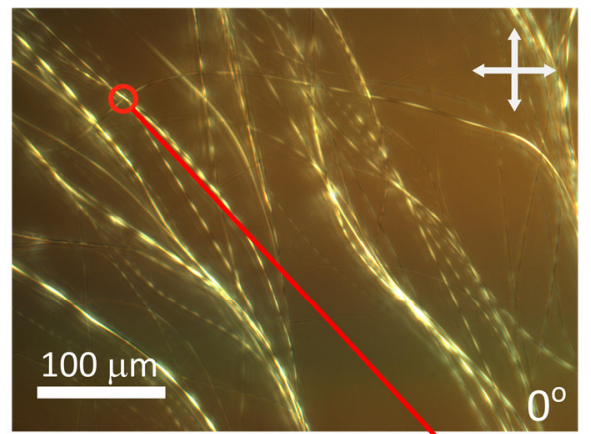

(c)

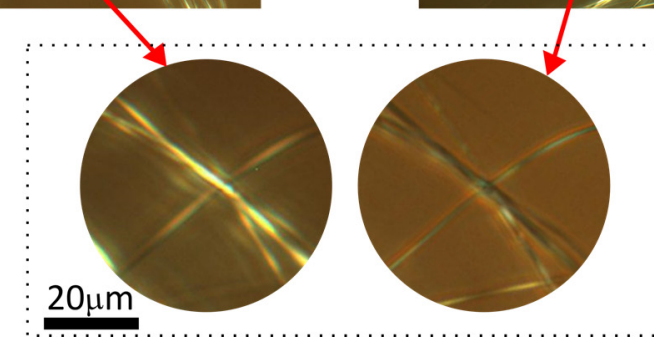

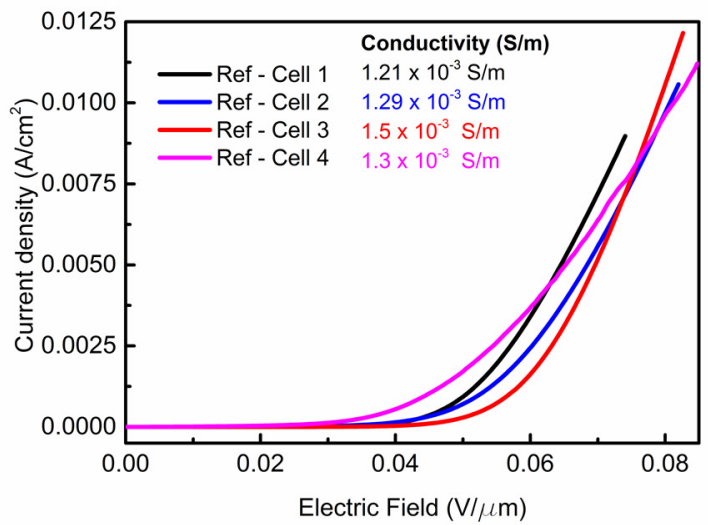

(b)

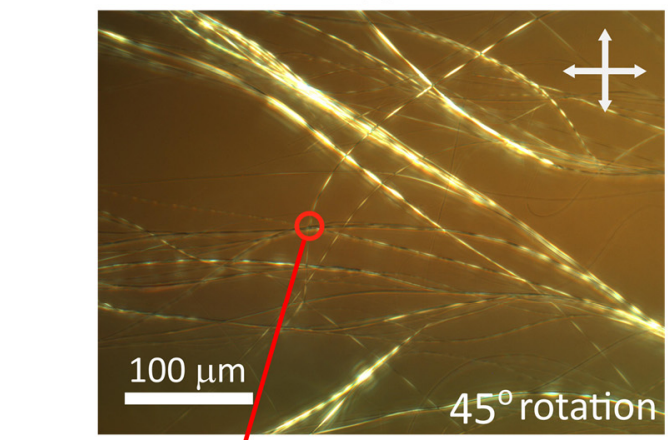

(d)

Figure 2. Conductivity measurements of liquid electrolyte in an ITO-ITO sandwich cell configuration with $25 \mu \mathrm{m}$ spacers for (a) HAT6 gelators or (b) reference electrolyte (inset in (a) illustrates test device schematic). Optical micrographs of Cell 3 from (a), under reflection at $25^{\circ} \mathrm{C}$ at angle (c) $0^{\circ}$ and followed by a $45^{\circ}$ rotation (d), and an enlarged region from both micrographs is compared. 


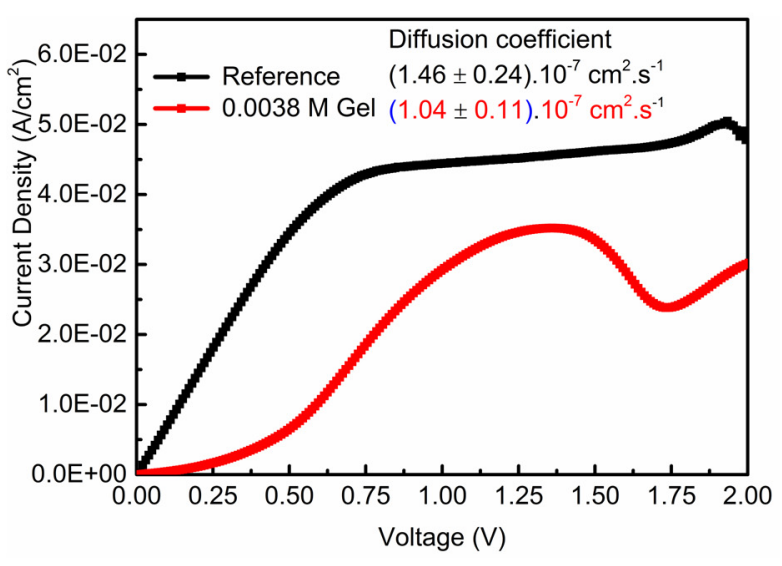

(a)

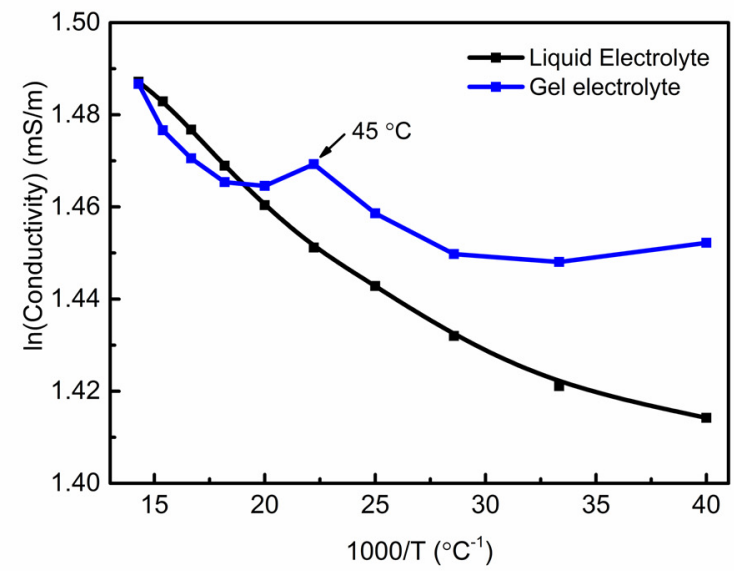

(b)

Figure 3. Linear sweep voltammetry (a) measurements performed at a scan rate of $10 \mathrm{mV} / \mathrm{s}$ to determine the diffusion coefficients of the ions in the reference and gel electrolytes in a FTO-FTO (platinum coated) sandwich cell configuration with $60 \mu \mathrm{m}$ spacers. (b) Variation of conductivity with in $25 \mu \mathrm{m}$ ITO-ITO cells at an applied voltage of $1 \mathrm{~V}$ at $1 \mathrm{KHz}$ frequency. 


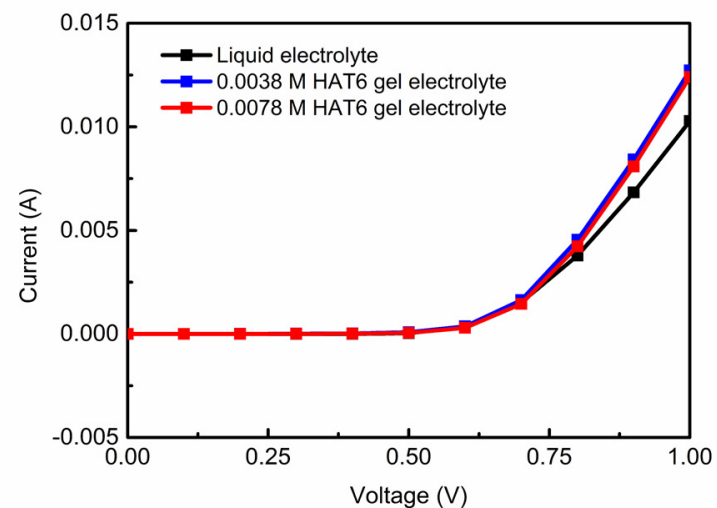

(a)

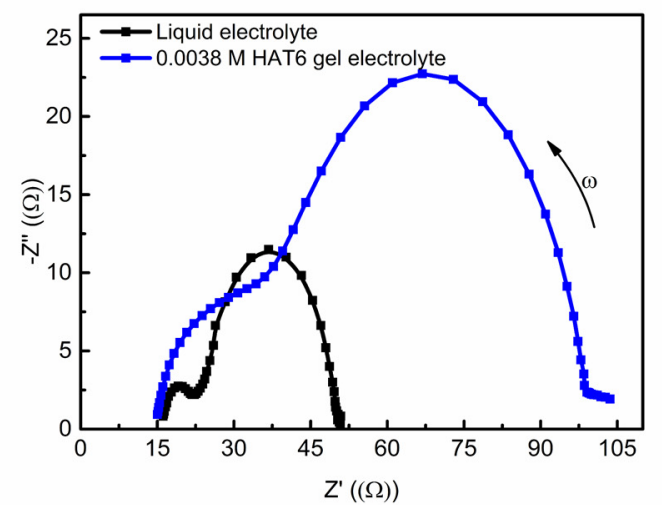

(c)

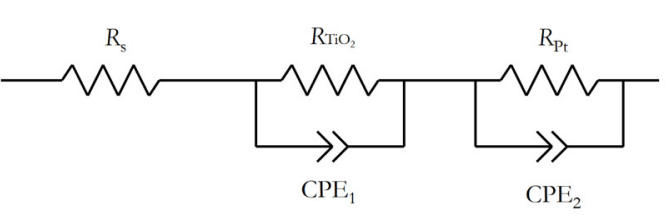

(e)

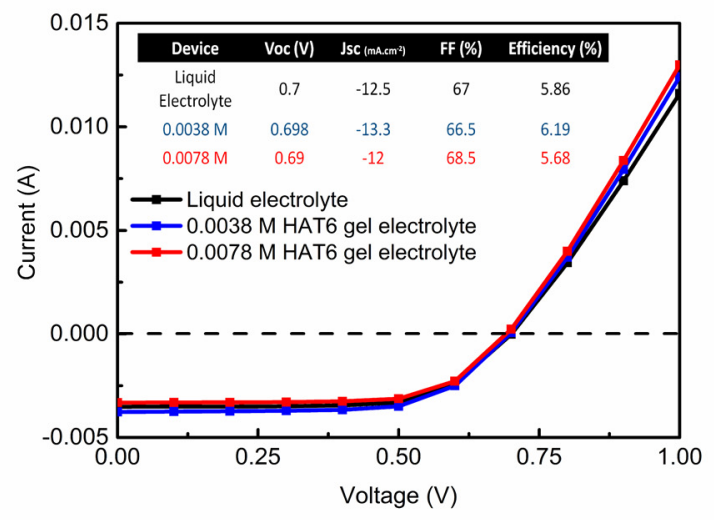

(b)

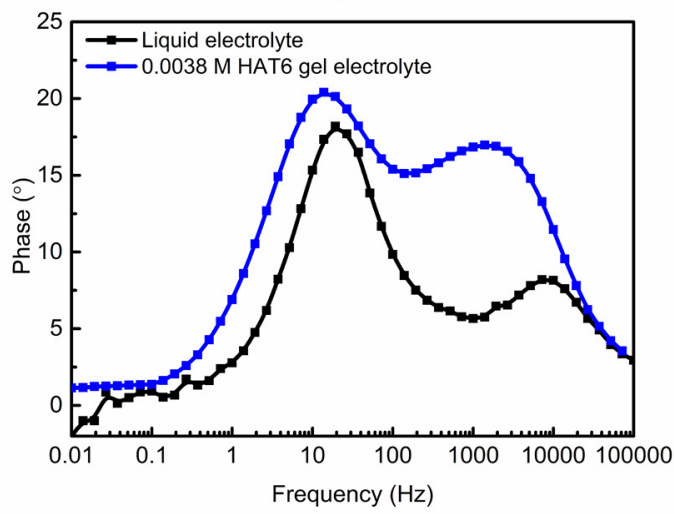

(d)

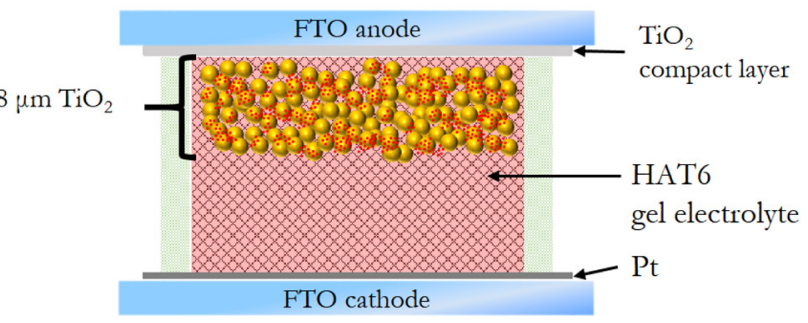

(f)

Figure 4. Compilation of solar cell performance using triphenylene-based gel electrolytes and their comparison with a standard liquid electrolyte. (a) Dark IV curves of DSSC devices filled with a reference liquid electrolyte and 0.0038 M, 0.0078 M HAT6 gel electrolytes. (b) Corresponding IV curves under simulated AM 1.5 light condition. (c) Nyquist plot obtained using EIS of a liquid and 0.0038 M HAT6 gel electrolyte in a DSSC configuration, at a biased voltage of -0.7 V under dark conditions. (d) Impedance angle as a function of frequency, using EIS presented in (c). (e) Equivalent circuit of the DSSC used for EIS measurements to model charge transfer parameters at various interfaces. (f) Schematic of the cross-section of a gel electrolyte DSSC device 


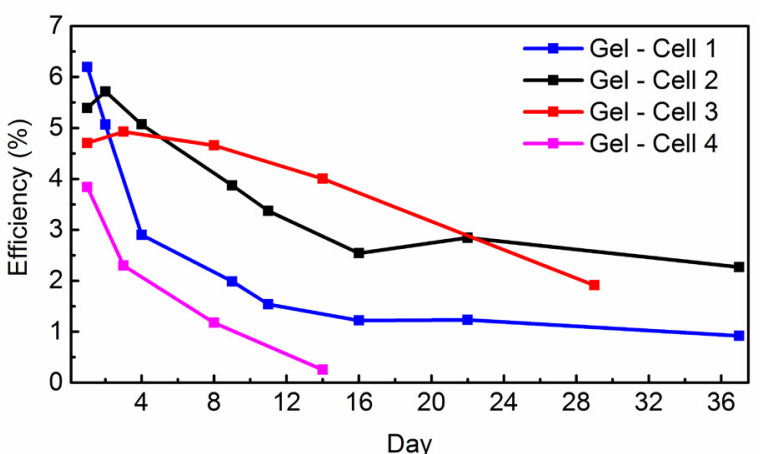

(a)

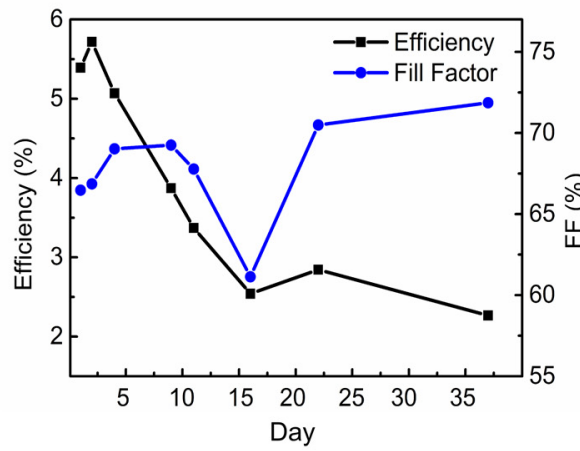

(c)

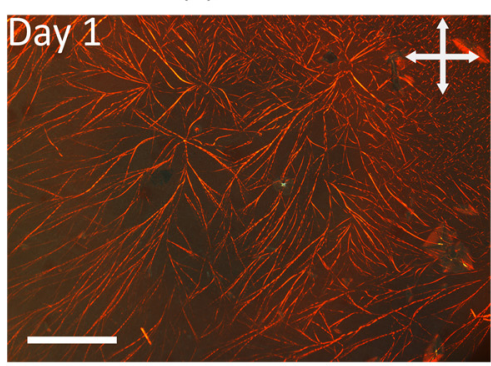

(e)

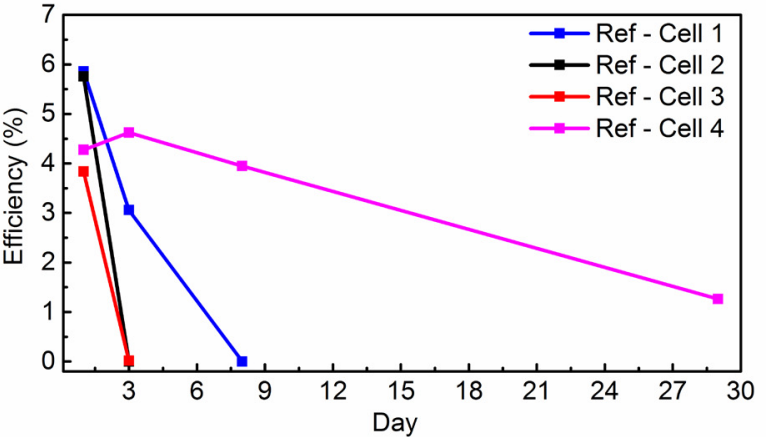

(b)

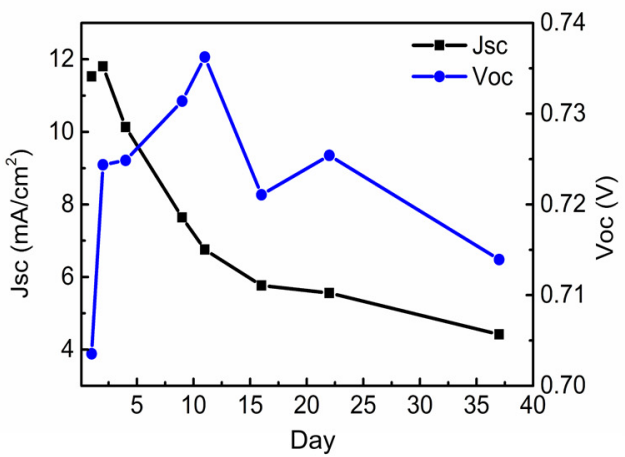

(d)

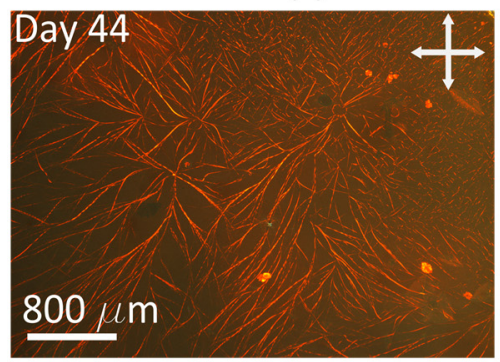

(f)

Figure 5. Variation of device efficiencies plotted against the number of days since fabrication (Day 1 is the day the devices were made). Data corresponds to devices utilising (a) 0.0038M HAT6 gel electrolytes is summarized in and (b) for cells utilizing refrence liquid electrolyte. (c),(d) Lifetime studies on a gel electrolyte solar cell (Cell 2 from Fig 4.a). Variation of individual performance parameters, fill factor and efficiency (c); Jsc and Voc (d) as a function of days since fabrication. Optical micrographs of the active area on (e) the $1^{\text {st }}$ and (f) the $44^{\text {th }}$ day, illustrating the stability of the gel texture. The scale bar in (e) and (f) is $800 \mu \mathrm{m}$. 